\title{
RESÍDUOS DE ANABOLIZANTES NA PRODUÇÃO ANIMAL: IMPORTÂNCIA E MÉTODOS DE DETECÇÃO
}

\author{
ANABOLIC RESIDUES IN LIVESTOCK PRODUTION: \\ RELEVANCY AND DETECTION METHODS
}

\author{
Keila Maria Roncato Duarte ${ }^{1,2}$ Fabiana Maria Siqueira Mariano da Silva ${ }^{1,3}$ \\ Cyro Ferreira Meirelles ${ }^{1,4}$
}

\section{- REVISÃO BIBLIOGRÁFICA -}

RESUMO

Com o desenvolvimento da indústria farmacêutica e o conseqüente surgimento de novas substâncias promotoras do crescimento animal, o setor agropecuário necessita de tecnologias cada vez mais eficientes e sensiveis para detectar possiveis resíduos deixados por este vasto número de substâncias anabolizantes, principalmente esteróides com atividades estrogênicas, androgênicas e progestogênicas. Tais substâncias, quando presentes na carne, gordura, ovos, entre outros, podem ser detectadas e quantificadas por uma série de métodos, dentre os quais, descrevemos os cromatográficos, baseados principalmente em cromatografia gasosa acoplada à espectrometria de massa (CG-EM), cromatografia líquida de alta eficiência (CLAE), cromatografia em camada delgada de alta eficiência (CCDAE) e os métodos imunológicos, como radioomunoensaio (RIA) e ensaio imunoenzimático (ELISA). Estes métodos têm como vantagens o elevado número de amostras que podem ser analisadas por período de tempo, aliadas à alta sensibilidade na detecção de resíduos.

Palavras-chave: métodos cromatogáficos, imunoensaios, hormônios, anabolizantes.

\section{SUMMARY}

With the development of the pharmaceutical industry, and consequently appearance of new substances which promote animal growth, the agricultural sector needs technologies more and more efficient and sensitive to detect possible residues left by this vast number of anabolic substances, mainly steroids with estrogenic, androgenic and progesterogenics activities. Such substances, when present in meat, fat, and eggs among others, can be detected and quantified by a series of methods, among the ones which we described the chromatographic methods, based mainly on gaseous chromatography coupled to the mass espectrometry (CG-MS), high performance liquid chromatography (HPLC), thin layer chromatography (HPTLC) and the immunological methods, as radioimmunoassay (RIA) and enzyme linked immunosorbent assay (ELISA). These methods have as advantages the high number of samples that can be analyzed by period of time, toghether with high sensibility and accuracy to detect anabolic residues.

Key words: immunoassays, hormones, chromatografic methods, anabolics.

\section{INTRODUÇÃO}

O crescimento cada vez maior da industrialização e a existência de um mercado cada vez mais globalizado vêm exigindo dos produtores rurais a utilização de modernas tecnologias ligadas à produção animal, cuja aplicação comprovadamente tem resultado em maior produtividade e melhor qualidade dos alimentos, entre eles o uso de anabolizantes, cujo uso é proibido no Brasil

\footnotetext{
${ }^{1}$ Laboratório de Endocrinologia, Centro de Energia Nuclear na Agricultura (CENA), Universidade de São Paulo (USP), Av. Centenário, 303, 13416-000, Piracicaba, SP.

${ }^{2}$ Engenheiro Agrônomo, Doutor em Microbiologia Agrícola. Pós-Doutorando do CENA, USP. E-mail: kmrduart@cena.usp.br. Autor para correspondência.

${ }^{3}$ Engenheiro Agrônomo, Mestre em Ciência e Tecnologia de Alimentos, Aluno de Doutorado do Curso Pós-Graduação, CENA, USP.

${ }^{4}$ Médico Veterinário, Professor Associado do CENA, USP.
} 
Compreende-se por anabolizantes, as substâncias que aumentam a retenção de nutrientes fornecidos pela alimentação, principalmente a retenção de nitrogênio protéico e não protéico e conseqüente transformação em proteína. Em última análise, aumento de peso e de massa muscular. Apesar dos resíduos deixados pelos anabolizantes em tecidos animais ser da ordem de partes por bilhão, sabe-se que a ação dos hormônios naturais ocorrem nesta ordem nos animais e neste caso, a quantidade não é fator relevante, e sim o simples fato de haver resíduo.

A regulamentação oficial para a utilização de hormônios em produção animal varia nos diversos países. Somente o dietilestilbestrol (DES) tem uso proibido em todos os países devido ao seu efeito carcinogênico (COLLINS et al., 1989).

Em 1989, a Comunidade Econômica Européia proibiu a utilização de substâncias anabolizantes para uso em animais, não permitindo a comercialização e a importação de carnes, que apresentem resíduos dessas substâncias (BOOTS et al., 1997). Em alguns países como Canadá, Austrália, Nova Zelândia, Argentina e Estados Unidos é permitido o uso dos compostos anabolizantes naturais tais como testosterona, progesterona, $17 \beta$-estradiol e dos sintéticos zeranol e acetato de trembolona (TBA). Nesses países, são controlados somente os resíduos dos compostos sintéticos com Limites Máximos de Resíduos (LMR) para o zeranol de $10 \mu \mathrm{g} \cdot \mathrm{kg}^{-1}$ e $2 \mu \mathrm{g} \cdot \mathrm{kg}^{-1}$ em fígado e músculo bovino respectivamente e de $1,4 \mu \mathrm{g} . \mathrm{kg}^{-1}$ de $\beta$-trembolona em tecido muscular bovino e de $14 \mu \mathrm{g} . \mathrm{kg}^{-1}$ de $\alpha$-trembolona em rim e fígado bovino, sendo que esses valores foram estabelecidos por um comitê misto da FAO/ OMS (Organização Mundial da Saúde) (COLLINS et al., 1989; LONE, 1997).

No Brasil, a partir de 24 de maio de 1991 (BRASIL, 1994) foi proibida a importação, produção, comercialização e uso de substâncias naturais ou artificiais, para fins de crescimento e/ou engorda de animais de abate, com permissão apenas para fins terapêuticos, sincronização de ciclo estral e preparação de doadores e receptores para a transferência de embriões (BRASIL, 1991).

Em 1995, foi determinado pelo Codex alimentarius, que os produtos 17b-estradiol, testosterona e progesterona seriam seguros à saúde assim como o acetato de trembolona e o zeranol desde que em doses inferiores a 2 e $10 \mathrm{mg} / \mathrm{kg}$ respectivamente. $\mathrm{O}$ dietiletilbestrol foi considerado como potencialmente tóxico (PALERMO-NETO, 1998).

\section{Agentes anabolizantes}

São substâncias usadas na produção animal, podendo serem descritos como biologicamente endógenos, compostos hormonais esteróides naturalmente presentes no organismo animal (testosterona, progesterona e estradiol 17- $\beta$ ) ou como biologicamente exógenos, dividindo-se em xenobióticos (acetato de trembolona -TBA, acetato de malengestrol -MGA e zeranol), esteróides sintéticos (etinilestradiol e metiltestosterona) e os estilbenos (dietilestilbestrol - DES e hexoestrol) (PATTERSON \& SALTER, 1985).

O mecanismo de ação dos anabolizantes se dá pela deposição de proteínas, que é resultado da diferença entre a síntese e a degradação protéica (LONE, 1997). Estudos conduzidos por HAYDEN et al. (1992) comprovaram um aumento na deposição de proteína sem a redução na degradação, indicando que os anabolizantes devem aumentar a síntese protéica. No entanto, estudos conduzidos por BUTTERY (1983) e SINNETT-SMITHet al. (1983) indicaram que os anabolizantes reduzem a degradação de proteínas. De acordo com HAYDEN et al. (1992), o tratamento com estradiol e acetato de trembolona separadamente ou em combinação não modifica os níveis de insulina, hormônio de crescimento (HC) ou cortisol. Por outro lado, compostos androgênicos reduzem a concentração de tiroxina (BUTTERY \& SINNETT-SMITH, 1984). Embora os anabolizantes não tenham um efeito direto sobre o hormônio de crescimento, eles potencializam a sua ação através de um aumento no número de receptores para hormônio de crescimento no fígado, podendo resultar em um aumento na concentração do fator de crescimento de fibroblastos (IGF-I) (JOHNSON et al., 1996; HANCOCK et al., 1991). Esta resposta parece ser potencializada ainda mais devido à ação do TBA em particular, por aumentar a resposta de células do músculo à ação de IGF-I, resultando em aumento na proliferação e diferenciação de células satélites (THOMPSON et al., 1989).

Os anabolizantes usados são aplicados como implantes, injeções oleosas ou como aditivos alimentares, sendo comum o emprego de "misturas" de anabolizantes, com dois ou mais agentes anabolizantes, com os quais se obtém um efeito sinergístico ou aditivo sobre o ganho de peso, permitindo-se a aplicação de doses menores de cada anabolizante, quando comparadas com os anabolizantes injetados isoladamente. Essa prática apresenta a vantagem de dificultar a detecção de resíduos nos tecidos, burlando com isso a fiscalização. 


\section{Resíduos de agentes anabólicos}

No mundo moderno, grande parte da segurança alimentar repousa no controle de remanescentes residuais nos alimentos, em decorrência do uso de pesticidas, ou drogas veterinárias, ou por acidentes envolvendo contaminantes ambientais. Resíduos de anabolizantes em carnes trazem sérios problemas de saúde pública. De acordo com EPSTEIN (1990), 3000 crianças em Porto Rico tiveram sérios problemas de desenvolvimento sexual prematuro e cistos ovarianos devido à ingestão de produtos cárneos com resíduos de zeranol.

Segundo o Comitê Misto do Codex Alimentarius/ FAO/ WHO, a ingestão de alimentos contaminados por anabolizantes pode levar ao aparecimento de distúrbios endócrinos como indução de puberdade precoce em crianças, avanços na idade óssea com repercussões negativas no crescimento, modificação dos caracteres sexuais, bem como cânceres principalmente no fígado e pâncreas (CARDOSO et al., 1999).

Os agentes anabólicos são geralmente metabolizados e seus metabólitos excretados ou levados pelos tecidos numa forma livre, biologicamente não ativa. O fígado converte os agentes anabólicos em metabólicos menos ativos e também em formas conjugadas, mais solúveis em água, que são secretadas na bile, sendo então excretados pelas fezes e urina. No entanto, uma parte dos metabólitos formados no fígado pode entrar no sistema circulatório e permanecer em outros tecidos como resíduos (HEITZMAN, 1983; RICO, 1983).

De acordo com ROSA \& DODE (1987), a via de administração mais utilizada em bovinos é o implante em parte não comestível da carcaça, como por exemplo, na orelha. A administração oral ou injeção (oleosa ou cristalina) aumenta a possibilidade de abusos e a ocorrência de resíduos em tecidos comestíveis, o que torna um problema de saúde pública. Os agentes anabólicos endógenos, quando usados na produção animal são rapidamente metabolizados pelo fígado, não sendo muito ativos quando administrados oralmente. Já os agentes anabólicos exógenos, ou sintéticos, são relativamente resistentes às biotransformações $e$, portanto, menos prontamente metabolizados no fígado, explicando com isso sua grande atividade quando administrados oralmente, favorecendo com isso seu acúmulo na musculatura, fígado e rins (NASCIMENTO, 1997).

Segundo HEITZMAN (1983), as maiores concentrações de resíduos de anabolizantes têm sido encontradas no local de administração, na bile, nas fezes e na urina. Concentrações medianas foram encontradas no fígado e nos rins e as menores concentrações nos músculos e gordura.

Os metabólitos do DES aparecem em três formas eletrofílicas: a) dienestrol; b) whidroxidienestrol, na forma glucoronil-conjugada e c) w-hidroxidienestriol, na forma sulfo-conjugada. $\mathrm{O}$ DES é excretado pelas fezes em uma forma livre e na urina como uma forma glucorona-conjugada. $\mathrm{O}$ fígado é o órgão que retém maior quantidade de resíduos de DES, de tal forma que até 60 dias após implantação ainda se encontra $0,1 \mu \mathrm{g} \cdot \mathrm{kg}^{-1}$ (RICO, 1983).

O zeranol e seus metabólitos são excretados na bile ou na urina após conjugação glucoronídea e/ou na forma de sulfato. Em mamíferos, os principais metabólitos do zeranol são zearalenona e taleranol, sendo esses metabólitos encontrados tanto nas fezes e urina como nos tecidos. A taxa desses metabólitos varia de acordo com a espécie. Em coelhos, o taleranol é o metabólito do zeranol (ERASMUSON et al., 1994; MIGDALOF et al., 1983).

O TBA, após ingerido, é rapidamente hidrolizado para $17 \beta$-trembolona, que é então convertido para $17 \alpha$-trembolona, o qual é considerado o mais abundante metabólito. Nos músculos, a maior quantidade de resíduos está na forma $17 \beta$-trembolona. Os resíduos solúveis estão por volta de $10 \%$ do total, sendo que o restante está ligado aos tecidos, isto é, não são extraídos por solventes orgânicos (POTTIER et al., 1975; RYAN \& HOFFMANN, 1978).

\section{Métodos para detecção de resíduos de agentes anabólicos}

A determinação de resíduos de agentes anabolizantes em material biológico é uma tarefa difícil. Para tal, os métodos utilizados, para serem considerados satisfatórios, deverão preencher uma série de requisitos entre os quais: ser adequado a uma rotina regular realizada por pessoa qualificada; permitir o manuseio de um grande número de amostras num pequeno espaço de tempo, para poder ser usado como um instrumento de fiscalização; ser seletivo para o composto, de modo a evitar falsos positivos; quantificar, de forma inequívoca, a concentração do composto presente; permitir tanto a quantificação do composto livre quanto seu conjugado; não ser muito oneroso, para não inviabilizar as análises de vigilância sanitária (PLAIZIER, 1993; EECKHOUT et al., 1998). 
Muitos métodos satisfazem alguns desses itens, mas nenhum satisfaz a todos. É possível, entretanto, conseguir resultados bastante satisfatórios com métodos que preencham a maioria desses requisitos. A determinação de resíduos de agentes anabolizantes em matrizes biológicas tais como urina, é difícil devido à contaminação com esteróides endógenos e componentes da dieta que interferem com a detecção de baixas concentrações.

Geralmente, para a detecção de resíduos de anabolizantes, dois tipos de métodos podem ser usados: (1) os imunoensaios tais como radioimunoensaio (RIA) e método imunoenzimático (ELISA), os quais são rotineiramente usados para detecção e (2) métodos físico-químicos, por cromatografia (CG-EM, CCDAE), os quais são usados para determinações quantitativas e como métodos de referência (HOFFMAN \& BLIETZ, 1983; LONE, 1997).

\section{Métodos imunoenzimáticos}

Na detecção de resíduos hormonais nos animais, metodologias diferentes podem ser utilizadas, como os imunoensaios do tipo "enzyme linked immunosorbent assay"- ELISA (HARLOW \& LANE, 1988; GODFREY, 1998) e radioimunoensaio - RIA.

$\mathrm{Na}$ década de 60, surgiu o RIA para quantificar hormônios em análises de bioquímica clínica de humanos e rapidamente passou a ser aplicado para determinação de resíduos de anabólicos devido à alta sensibilidade, precisão e repetibilidade. Contudo, existem desvantagens desta metodologia, como o uso de material radioativo e conseqüentemente, pessoal treinado e laboratório diferenciado (MATOS, 1996). Estudos recentes (WIENER et al., 2000) mostraram que radioimunoensaios têm sido vastamente utilizados para monitoramento de hormônios, sejam eles naturais, para controle de ciclos estrais, ou administrados, para finalidade de aumento de peso. Estes imunoensaios apresentam rapidez, alta sensibilidade e grande precisão na determinação de hormônios em gado bovino.

Os imunoensaios baseiam-se na reação antígeno-anticorpo e posterior revelação desta reação, de forma a quantificar e qualificar o ensaio. A revelação pode ser por reação enzimática (ELISA) ou por emissão de radioatividade, no caso de um isótopo marcado (RIA) (HARLOW \& LANE, 1988). Em ambos os casos, é necessário que se produza o anticorpo contra a molécula que se deseja identificar ou detectar. Os anticorpos produzidos podem ser policlonais, quando se trata de soro de coelho ou galinha, ou ainda monoclonais, quando produzidos por fusão de baço de camundongo e células de mieloma, para a formação de hibridomas e posterior seleção dos clones (KÖHLER \& MILSTEIN, 1975).

O uso de anticorpos monoclonais para estes ensaios de detecção de anabolizantes tem sido realizado para diagnóstico de diversos anabolizantes por apresentar resultados rápidos e confiáveis, detectando níveis ao redor de $0,05 \mathrm{ppm}$ das substâncias monensinas, oxitetraciclinas, trembolona, zeranol, carbadox, closantel, flubendazole ivermectina e clembuterol em animais como galinhas, perus, bovinos e caprinos (WATANABE $\boldsymbol{e t} \boldsymbol{a l}$. , 1998).

A identificação de beta-agonistas como clenbuterol, tem sido realizada utilizando radioimunoensaios (RIA), com anticorpos policlonais. Os beta-agonistas estão sendo utilizados ilegalmente como promotores de crescimento em gado bovino e em cavalos e sua identificação faz-se necessária. No trabalho de RODA et al. (2000), um teste rápido e de alta sensibilidade foi desenvolvido para determinação de clenbuterol em urina de bovinos, baseados em RIA, mostrando-se eficiente na detecção de 0,5 a 1,2ppb de clenbuterol.

$\mathrm{O}$ uso do acetato de trembolona (TBA) tem sido monitorado em amostras de origem animal, principalmente na urina de animais, utilizando-se a cromatografia gasosa acoplada a espectrometria de massa (CG-EM) para purificação e identificação aliado a imunensaios do tipo ELISA (STERK $\boldsymbol{e t}$ al., 1998).

Em trabalho recente, CARDOSO et al. (1999) analisaram fígado bovino colhido de matadouros-frigoríficos inspecionados pelo Serviço de Inspeção Federal (SIF), participantes da lista de exportadores e verificaram a ausência de resíduos de dietilestilbestrol (DES) encontrando, porém, resíduos de zeranol, na frequência de $0,52 \%$ das amostras analisadas por CG-EM. Dados conflitantes foram encontrados quando se utilizava RIA. Cerca de $15,6 \%$ das amostras apresentaram contaminação por zeranol e 2, $16 \%$ para DES.

\section{Métodos cromatográficos}

Durante os últimos anos, tem havido uma tendência de uso de métodos cromatográficos, como cromatografia líquida de alta eficiência (CLAE), cromatografia gasosa acoplada à espectrometria de massa (CG - EM) e cromatografia em camada delgada de alta eficiência (CCDAF), como importantes ferramentas para a análise de esteróides anabolizantes em geral. 
A técnica do CG-EM é uma das mais importantes na identificação de anabolizantes, visto que combina o poder de resolução do cromatógrafo a gás à alta seletividade do detector de massa, tendose com isso a identificação de anabolizantes em concentrações muito baixas (ng. $\ell^{-1}$ ou $\mathrm{ng} \cdot \mathrm{kg}^{-1}$ ) (SAEED et al., 1999).

Alguns dos esteróides anabolizantes e muitos de seus metabólitos não exibem bom comportamento cromatográfico, principalmente devido a grupos hidroxilas ou cetônicos em sua estrutura, sendo necessária, na análise da maioria dos esteróides por cromatografia a gás, uma prévia derivação, diminuindo a instabilidade ao calor apresentada pelos anabolizantes MARCHAND et al., 2000).

Pela derivatização, introduz-se na molécula do anabolizantes um grupo de alto peso molecular, propiciando maior seletividade e especificidade à técnica cromatográfica (SEGURA et al., 1998).

Entre uma grande quantidade de reagentes descritos para a derivatização dos grupos hidroxilas dos anabolizantes, a trimetilsilização tem sido particularmente vantajosa. Metilsilitriflouroacetamida (MSTFA) ou bistrimetilsililtrifluoro acetamida (BSTFA) têm sido os reagentes preferencialmente utilizados. No entanto, os álcoois terciários não são facilmente derivatizados somente com esses reagentes (AYOTTEet al., 1996). Considerando o fato de que muitos anabolizantes apresentam um grupo terciário 17 $\beta$-hidroxi, a presença de um catalizador é necessária para derivatizar completamente estes compostos. O TMSI é considerado o melhor catalisador (SEGURA et al., 1998).

Em trabalho realizado por TUINSTRA $\boldsymbol{e t}$ al. (1983), a cromatografia gasosa acoplada à espectrometria de massa (CG-EM) mostrou ser um método sensível e preciso para a determinação de resíduos de DES em urina bovina. O CG-EM tem sido utilizado em diversos ensaios na análise de metabólitos em fezes, urina e tecidos de bovinos para monitoramento do uso de anabolizantes no rebanho (PUYMBROECK $\boldsymbol{e t}$ al., 1998).

De acordo com MATTOS (1996), a espectrometria de massa é uma radiografia da molécula, representando os fragmentos da massa que se obtém de um determinado composto, após ser submetido a um impacto eletrônico.

Vários métodos de cromatografia em camada delgada foram desenvolvidos para determinar anabólicos na urina, nas fezes e nas rações destinadas à alimentação de animais. Aplicam-se também na pesquisa de resíduos em tecidos. Das várias técnicas, a que possui sensibilidade suficiente para determinar resíduos é a cromatografia em placa de alta eficiência (DELAHAUT et al., 1990).

De acordo com DE BRABANDER $\boldsymbol{e t}$ al. (1989), a utilização de cromatografia em camada delgada de alta eficiência (CCDAE) permitiu uma determinação rotineira de vários resíduos anabólicos em amostras de urina bovina, com sensibilidade na faixa de 0,5 para $10 \mu \mathrm{g} \cdot \mathrm{kg}^{-1}$. O uso de CCDAF tem a capacidade de separar e analisar um grande número de compostos em poucos minutos e com alta resolução, detectando anabolizantes na ordem de ppb (partes por bilhão) (LONE, 1997).

A cromatografia líquida de alta eficiência (CLAE) acoplada a detectores de ultra-violeta (UV), nas análises de resíduos de anabolizantes, tem sido usada como método preparativo, ou seja, para purificação das amostras biológicas para posterior aplicação de técnicas mais sensíveis de detecção e quantificação, como CG-EM, CCDAF, ELISA, RIA (DAESELEIRE $\boldsymbol{e t}$ al., 1991; DAESELEIRE $\boldsymbol{e t}$ al., 1992).

Atualmente tem sido desenvolvidos detectores de UV mais sensíveis para serem acoplados à CLAE, como o detector por arranjo de fotodiodos (DAD), o qual possibilita a detecção e quantificação de resíduos de anabolizantes em amostras biológicas. KOOLE et al. (1999) descreveram um método de CLAE -DAD para análise e identificação de 20 substâncias com propriedades anabolizantes em amostras de urina com limite de detecção de 5 - $10 \mathrm{ng} . \mathrm{g}^{-1}$ para diferentes substâncias.

Outro tipo de detector que está sendo desenvolvido para ser acoplado a CLAE é aquele por espectrometria de massa (EM). A CLAE - EM é um método de detecção e identificação, com baixo limite de detecção, tendo a vantagem em relação ao CG-EM por não haver necessidade de derivatização das amostras, diminuindo custos e possíveis perdas que ocorrem nessa etapa.

HORIE \& NAKAZAWA (2000) utilizaram CLAE - EM para a determinação de metabólitos de trembolona e zeranol em amostras de fígado e músculo com limite de detecção de $0,5 \mathrm{ng} \cdot \mathrm{g}^{-1}$. De acordo com os autores a principal vantagem desse tipo de método para detecção de resíduos de anabolizantes é que as amostras não precisam passar por uma etapa de derivatização, evitando perdas indesejáveis.

\section{CONCLUSÃO}

$\mathrm{O}$ acetato de trembolona (TBA) e o zeranol são proibido no Brasil, mas são permitidos nos Estados Unidos, México, Colômbia e Argentina. Assim, o DES é contrabandeado via Paraguai 
(PALERMO NETO, 1998). De acordo com a fiscalização brasileira (BRASIL, 1994), das 50 milhões de cabeças bovinas abatidas em 1994, apenas 800 foram inspecionadas, ou seja, somente a carne para exportação foi analisada. O Ministério da Agricultura e do Abastecimento adota a cromatografia gasosa acoplada à espectrometria de massa para detecção de anabolizantes e especificamente o RIA para resíduos de dietilestilbestrol (DES) e zeranol, uma vez que o limite de tolerância de qualquer destas substâncias é zero, já que o uso é proibido (CARDOSO et al., 1999). Esta baixa porcentagem de cabeças bovinas analisadas é devido à falta de laboratórios credenciados e ao fato de que os imunoensaios (ELISA e RIA), apesar de práticos e de alta sensibilidade, tornam-se inviáveis devido ao custo dos reagentes e dos "kits" que são importados.

Este trabalho tem, portanto, o intuito de alertar sobre o problema do uso de anabolizantes de forma ilegal no Brasil, bem como comentar sobre metodologias eficientes utilizadas na detecção de resíduos, de forma a contribuir para a qualidade da carne encontrada principalmente no mercado interno.

\section{REFERÊNCIAS BIBLIOGRÁFICAS}

AYOETTE, C.; GOUDREAULT, A.; CHARLEBOIS, J. Testing for natural and synthetic anabolic agents in human urine. Journal of Chromatography B, v.687, p.03-25, 1996.

BOOTS, R., ANDERSON, P, DEHAAN, K. Growth stimulants: compounds, concentrations, conbinations and regulations. In IMPACT AND CARCASS VALUE OF BEEF CATTLE SYMPOSIUM, 1997, Oklahoma. Proceedings... Oklahoma : Oklahoma State University, 1997. p.10-14.

BRASIL. MAARA. Relatório apresentado pelos membros da comissão nominada pelo Ministério da Agricultura, do Abastecimento e Reforma Agrária através da portaria no. 51 sobre o uso de promotores do crescimento hormonal em pecuária de corte. Brasília, 1994. 130p.

BRASIL. Ministério da Aghricultura. Portaria $\mathrm{n}^{0} 51$ de 24 de maio de 1991. Diário Oficial, Brasília, 27 de maio de 1991. Seção I. p.9989.

BUTTERY, P.J. Hormonal control of protein deposition in animals. Proceedings of Nutrition Society, v.42, n.2, p.137148, 1983.

BUTTERY, P.J., SINNETT-SMITH, P.A. The mode of action of anabolic agents with special reference to their effects on protein metabolism - some speculations. In: ROCHE, J.F., O'CALLAGAN, D. Manipulation of growth in farm animals. London : Elsevier Applied Science, 1984. Chap.4, p.211-234.

CARDOSO, O.M.C.; SILVA, T.J.P.; SANTOS, W.L.M. Ocorrência de resíduos de dietilestilbestrol e zeranol em fígado de bovinos abatidos no Brasil. Ciência e Tecnologia de Alimentos, v.19, n.3, p.1001-1014, 1999.
COLLINS, S.S., BELK, K.E., CROSS, H.R.. The EEC ban against growth-promoting hormones. Nutrition Reviews, v.47, n.8, p.238-246, 1989.

DAESELEIRE, E.A.I., DE GUESQUIÈRE, A. VAN PETEGHEM, C.H. Combined high-performance chromatography and radioimmunoassay for screening of 19nortestosterone and methyltestosterone residues in meat samples. Journal of Chromatography, v.564, n.2, p.445449, 1991.

DAESELEIRE, E.A.I., DE GUESQUIÈRE, A., VAN PETEGHEM, C.H. Multiresidue Analysis of anabolic agents in muscle tissues and urines of cattle by CG-MS. Journal of Chromatographic Science, v.30, n.10, p.409414, 1992.

DE BRABANDER, H.F.; VANHEE, P.; VAN HOYE, S.; $\boldsymbol{e}$ a $\boldsymbol{a}$. Improved chromatographic clean-up of anabolics in bovine urine. Journal of Planar Chromatography, v.2, n.2, p.33$38,1989$.

DELAHAUT P., DUBOIS, M., MAGHUIN-ROGISTER, G. Androgènes anabolisants de synthèse dans les matières fécales des bovins: dosage radio-immunologique après purification par Chromatographie liquide à haute performance. Annales de Recherches Veterinaires, v.21, n.1, p.13-22, 1990.

EECKHOUT, N.J. van, PETEGHEM, C.H. van, HELBO, V.C. New database on hormone and veterinary drug residue determination in animal products. The Analyst, v.123, n.12, p.2423-2427, 1998 .

EPSTEIN, S.S. Chemical additives in beef industry. Section on environmental health policy. International Journal of Health Services, v.20, n.2, p.277-280, 1990.

ERASMUSON, A.F.; SCAHILL, B.G.; WEST, D.M. Natural zeranol (alpha-zearalanol) in the urine of pasture-fed animals. Journal of Agricultural and Food Chemistry, v.42, n.12, p.2721-2725, 1994.

GODFREY, M.A.L. Immunoaffinity extraction in veterinary residue analysis - a regulatory viewpoint. The Analyst, v.123, n.12, p.2501-2506, 1998 .

HANCOCK, D.L., WAGNER, J.F., ANDERSON, D.B. Effects of strogen and androgens on animal growth. In: PEARSON, A.J., DUTSON, T.R. (eds). Growth regulation in farm animals: advances in meat research. London: Elsevier Applied Science, 1991. Chap. 7, p.255-297.

HARLOW, E., LANE, D. Antibodies: a laboratory manual. New York : Cold Spring Harbor Laboratory, 1988. 726p.

HAYDEN, J.M., BERGEN, W.G., MERKEL, R.A Skeletal muscle protein metabolism and serum growth hormone, insulin and cortisol concentrations in growing steers implanted. with estradiol $17 \mathrm{~b}$, trembolone acetato, or estradiol-17b plus tre. with estradiol $17 \mathrm{~b}$, trembolone acetato, or estradiol-17b plus trenbolone acetate. Journal of Animal Science, v.70, n.7, p.2109-2119, 1992.

HEITZMAN, R.J. Coordination of information on residues of veterinary drugs in the European Comunity. Analytica Chimica Acta, v.275, p.17-22, 1983.

HOFFMAN, B., BLIETZ, C. Application of radioimmunoassay (RIA) for the determination of residues of anabolic sex hormones. Journal of Animal Science, v.57, n.1, p.239-246, 1983. 
HORIE, M, NAKAZAWA, H. Determination of trenbolone and zeranol in bovine muscle and liver by liquid chromatography - electrospry mass spectrometry.

Journal of Chromatography A, v.882, p.53-62, 2000.

JOHNSON, B.J., ANDERSON, P.T., MEISKE, J.C., et al. Effects of a conbined trenbolone acetate and estradiol implant on feedlot performance, carcass characteristics and carcass composition of feedlot steers. Journal of Animal Science, v.74, n.2, p.363-371, 1996.

KÖHLER, M., MILSTEIN,C. Continuous cultures of fused cells secreting antibody of predifined specificity. Nature, v.256, p.495-497, 1975.

KOOLE, A., FRANKE, J.P., ZEEUH, A.A. Multi-residue analysis of anabolics in calf urine using highperformance liquid chromatography with diode-array detection. Journal of Chromatography B., v.724, p.41-51, 1999 .

LONE, K.P. Natural Sex steroids and their xenobiotic analogs in animal production: growth, carcass quality, pharmacokinetics, metabolism, mode of action, residues, methods and epidemiology. Critical Review in Food Science and Nutrition, v.37, n.2, p.93-209, 1997.

MARCHAND, P.; BIZEC, B.; GADE, C., et al. Ultra trace detection of a wide range of anabolic steroids in meat by gas chromatography coupled to mass spectrometry. Journal of Chromatography A., v.867, p.219-233, 2000.

MATOS, S. Controle de resíduos anabólicos em carnes. Revista Nacional da Carne, n.227, p.44-48, 1996.

MIGDALOF, B.H., DUGGER, H.A., HEIDER, J.G., et al. Biotransformation of zeranol disposition and metabolism in female rat, rabbit, monkey and man. Xenobiotica, v.13, n.4, p.209-221, 1983.

NASCIMENTO, E. Importância do controle de resíduos de agentes anabolizantes em carnes. Campinas : Instituto de Tecnologia de Alimentos - Centro de Tecnologia de Carnes. 1997. (Apontamentos de curso).

PALERMO NETO, J. Anabolizante e pecuária de corte. Revista de Educação Continuada do CRMV - SP, v.1, n.1, p.10-15, 1998.

PATTERSON, R.L., SALTER, L.J. Anabolic agents and meat quality: a review. Meat Science, v.14, n.4, p.191-220, 1985.

PLAIZIER, J.C.B. Validation of the FAO/IAEA RIA kit for the measurament of progesterone in skim milk and blood plasma. Viena : IAEA-TECDOC - 708, june 1993, p.151156.

POTTIER, J.; BUSIGNY, M.; GRANDADAM, J.A. Plasma kinetics, excretion in milk and tissue levels in the cow following implantation of trenbolone acetate. Journal of Animal Science, v.41, n.3, p.962-968, 1975.

PUYMBROECK,U.M. van, LEYSSENS, L., VANDERZANDE, D., et al. Metabolites in feces can be important markers for the abuse of anabolic steroids in cattle. The Analyst, v.123, n.12, p.2449-2452, 1998.
RICO, A.G. Metabolism of endogenous and exogenous anabolic agents in cattle. Journal of Animal Science, v.57, n.1, p.226-232, 1983.

RODA, A., MANETTA, A.C., PIAZZA, F. A rapid and sensitive 384-microtiter wells format chemiluminescent enzyme immunoassay for clenbuterol. Talanta, v.52, p.311-318, 2000.

ROSA, G.O., DODE, M.A.N. Hormônios anabolizantes revisão. Revista Criadores, v.56; n.686, p.129-137, 1987.

RYAN, J.J., HOFFMANN, B. Trenbolone acetate: experience with bound residues in cattle tissues Journal Association of Official Analytical Chemists, v.61, n.5, p.1274-1279, 1978.

SAEED, T.; ISMAEL, N.; AHMAD, N., et al. Assessment of the levels of anabolic compounds in Kuwait meat industry: optimization of a multiresidue method and the results of a preliminary survey. Food Control, v.10, p.169-174, 1999.

SEGURA, J.; VENTURA, R.; JURADO, C. Derivatization procedures for gas chromatographic-mass spectrometric determination of xenobiotics in biological samples, with special attention to drugs of abuse and doping agents. Journal of Chromatography B, v.713, p.61-90, 1998.

SINNETT-SMITH, P.A., DUMELOW, N.W., BUTTERY, P.J. Effects of trenbolone acetate and zeranol on protein metabolism in male castrate and female lambs. British Journal of Nutrition, v.50, n.2, p.225-234, 1983.

STERK, S., Van TRICHT, F., LE, B., et al. Production and stability testing of incurred refernce materials for the anabolic steroid trenbolone in bovine urine. The Analyst, v.123, n.12, p.2563-2566, 1998.

THOMPSON, S.H., BOXHORN, L.K., KONG, W., et al. Trenbolone alters the responsiveness of skeletal muscle satellite cells to fibroblast growth factor and insulin-like growth factor I. Endocrinology, v.124, n.5, p.2110-2117, 1989.

TUINSTRA, L.G.M.Th., TRAAG, W.A., KEUKENS, H.J., et al. Procedure for the gas chromatographic-mass spectrometric confirmation of some exogenous growth-promoting compounds in the urine of cattle. Journal Chromatographic, v.279, n.11, p.533-542, 1983.

WATANABE, H., SATAKE, A., MATSUMOTO, M., $\boldsymbol{e} \boldsymbol{t} \boldsymbol{a}$. Monoclonal based enzyme-linked immunososbent assay and immunochromatographic rapid assay for monensin. The Analyst, v.123, n.12, p.2573-2578, 1998.

WIENER,P., ANGUS, K.D., McCULLOUGH, E. Growth hormone response to infusion of somatostatin in dairy calves: testing itsuse as a diagnostic tool for genetic merit. Animal Science, v.71, p.219-226, 2000. 